\title{
The management of anorexia nervosa
}

\author{
R. L. Palmer
}

The severity of anorexia nervosa can vary from mild to life threatening. It is sometimes transient but often chronic. Such variety of disorder requires variety of response. The clinician must choose the right treatment to offer at the right time. The literature contains plenty of advice but most of this is based upon experience and opinion rather than on systematic research and treatment trials. For the most part, this paper will be no exception. Anorexia nervosa is a disorder which is distinct from other psychiatric syndromes but is of uncertain cause. In the face of this uncertainty, treatment tends to be informed by the favoured formulation of the clinician, usually some sort of 'multifactorial theory'. Again this paper is no exception. It will concentrate upon the management of anorexia nervosa in late adolescence and adulthood. The treatment of children requires a different approach (Lask \& Bryant-Waugh, 1993). The emphasis of the paper will be upon what can go wrong as well as what may be the best interventions to offer. Often the things that go wrong have more to do with the context of treatment and the way in which it is offered rather than with the treatment intervention itself.

\section{The nature of the problem}

The core features of anorexia nervosa are well known and the criteria set out in ICD-10 and DSMIV are closely similar for practical purposes. Although atypical cases are not uncommon, making the diagnosis is not usually difficult. When there is real doubt the diagnostic puzzle can be tough and is worthy of a paper in its own right. What is not so easy is the development of a 'feel' for what eating disorders are about. In this respect it may be useful to think of anorexia nervosa as involving an entanglement of two sets of ideas and the consequences of that entanglement. One set of ideas concerns weight and eating control. The other concerns wider personal issues such as self-esteem, emotional control and self-image in the broadest sense. These issues are mixed up for many people in our society, especially for young women.

The idea that self-worth may be enhanced by slenderness is often, although not always, the initial motivation for the eating restraint which seems to be the necessary way into anorexia nervosa. Most slimmers give up restraint in the face of their bodies' regulatory responses. This is the arguably healthy 'what the hell; it's not worth it' response. For some reason the anorectic-to-be persists and becomes caught up in what seems like a nest of vicious circles. For whatever reason, the more she tries to control her eating and weight, the more potentially out of control these issues feel and the more frightening seems the option of, in any sense, letting go. Her ideas become more extreme. She feels worse about herself. She comes to feel like the driver of a car who is stuck with her foot flat down on both the accelerator and the brake. Too often it feels as though everyone else just wants to give her a push. Of course, she needs to get moving but simply pushing may make matters worse.

\section{Assessment and engagement}

The assessment involves more than just making the diagnosis. This paper assumes that a diagnosis has been made and will discuss what management may appropriately follow. However, management begins within the context of the assessment interviews. It is important to remember that the patient is assessing the clinician every bit as much as the clinician is assessing her (or occasionally him). Does the clinician seem knowledgeable, confident and trustworthy? Does she feel understood? Does she feel safer or more threatened as a result of the interview? How things proceed may

R. L. Palmer, FRCPsych, is a senior lecturer in psychiatry at the University of Leicester and a honorary consultant psychiatrist at Leicester Mental Health Services NHS Trust. He is the consultant to the Leicester Eating Disorders Service based at the Leicester General Hospital, Gwendolen Road, Leicester LE5 4PW. He is the author of Anorexia Nervosa; A Guide for Sufferers and their Families (Penguin). 
depend on the patient's answers to these questions. The conclusions which she reaches are likely to be influenced by the way in which the assessment is conducted.

Anorexia nervosa is a disorder of fearful mixed feelings. Its management needs to take this into account from the beginning. Personally, I always start off the first assessment interview by asking the patient what she feels about coming along. This gives an opportunity for the expression of what are usually quite complicated feelings about the consultation. If the patient expresses only clear antipathy or resentment at having been pushed by others into coming along, I offer her the opportunity of opting out of the consultation. However, in several hundred such first interviews, only one sufferer has ever taken up my offer and she returned within a few weeks. Making the offer can change the nature of the interview and at least creates an early chance to explore any hostility and ambivalence.

\section{Diagnostic issues}

Apart from the diagnosis of the eating disorder itself, there is a need to consider both Axes I and II comorbidity. This does not involve much that is out of the ordinary. The important thing is to make the usual mental state examination even if the main diagnosis is evident and unequivocal. However, assessment of the more specific psychopathology and behaviours does require the clinician to ask questions which would not be routinely explored in such detail. Likewise, the usual history should be supplemented with greater attention to issues of weight and eating even before the onset of frank disorder (Box 1).

\section{Assessment of special issues}

Some issues which require special attention in the assessment of someone with anorexia nervosa are:

(a) Lifetime weight history, especially highest ever and premorbid stable weight. Premorbidly obese subjects may have difficulty sustaining an average weight without undue restraint. There may be no premorbid stable weight in an individual who has been eatingdisordered (or has been slimming) since their teenage years. It is useful to ask what weight the person would prefer to be if they had a magic wand which erased problems of weight control.

(b) History of eating restraint. (c) Use of abnormal weight control methods: vomiting, laxatives, diuretics, etc.

(d) Presence or absence of bingeing. Full bingeing involves eating larger quantities of food than would be expected in the particular circumstances and with a subjective sense of loss of control. Sometimes anorexia nervosa sufferers report any lapse from their habitual tight control as a binge.

(e) Ideas of loss of control of weight and eating.

(f) Disturbance of body image.

(g) Use of exercise as a method of attempted weight control.

(h) Beliefs about weight control and 'healthy eating'.

Various questions and standardised interviews are available for the assessment of these kind of issues. However, they do not replace the clinical interview in practice. A brief review of such methods can be found in Garner (1995).

While listening to the history it is almost impossible not to entertain ideas as to why the patient has developed an eating disorder. How has weight and eating control come to be so tangled up with the person's sense of self-worth and selfcontrol? What has undermined her self-esteem? Why has this person developed this illness at this time? What has it been like to be this person and to lead her life? And so on. It is important to think up possible answers to such questions. For instance, a history of childhood sexual abuse may be relevant. It is even more important to keep an open mind and not to be too convinced that one has the real answers. It is useful to generate hypotheses - or stories - as this can aid understanding and empathy. But to elevate your favoured story to the status of 'the truth' about the matter is to risk being damagingly wrong.

It may often be useful to seek a collateral history from the patients' family or some other relevant informant. Some authorities frame the whole assessment within a family interview on the

\section{Box 1. Diagnostic issues}

Assessment should include attention to:

Diagnosis of the eating disorder

Diagnosis of Axis I comorbidity

Diagnosis of Axis II comorbidity - especially obsessional traits or borderline personality disorder

Detection of physical complications Description of the context of the illness What 'story' can be told about the illness? 
grounds that anorexia nervosa is best viewed as a product of a derailed adolescent development within the family (Crisp, 1980). While there is much to be said for this view, it is not always possible to work in this way. For instance, the patient may clearly want to present herself alone. Issues of separation and individuation may be central and an important focus. However, these can be addressed in relation to the joint decision of the patient and the clinician as to whether to involve the family in the assessment and subsequent therapy.

Assessment needs to include the physical state of the patient. An obvious aspect of this is the act of weighing the patient. This may seem to be a simple matter. Often it is. Sometimes, however, the patient is reluctant to be weighed or even refuses. This faces the clinician with a dilemma. Is it better to push the matter and risk seeming to make weight 'too important', or worse, getting into a fight? Or is going along with not weighing the patient not only missing out on gaining important information, but also somehow unhelpfully endorsing the patients' fearful avoidance? A way forward is to use the situation as an opportunity to explore the patient's feelings and ideas about weight and the dilemmas which she is facing. Framed in this way the act of weighing can come to be a part of both assessment and engagement, perhaps even of therapy.

\section{Physical assessment}

Anorexia nervosa has a significant mortality from physical complications as well as from suicide. Initial assessment should include the physical state. Furthermore, there is a need for continued monitoring throughout treatment. There are many potential complications. The following are some pieces of general advice:

(a) Sufferers who are at a very low weight (BMI $<15)$, feel weak or complain of physical symptoms should usually be examined and have an ECG as part of their initial assessment.

(b) Blood tests are indicated for almost all patients. Electrolyte disturbance, especially hypokalaemia, and dehydration are common in sufferers who vomit or abuse laxatives or diuretics. Potassium supplements may be required as a first aid measure. Anaemia may occur. Moderate leucopenia is common and tends to remit with weight gain. Plasma proteins may be low.

(c) The results of the physical assessment should be discussed with the patient. Most of the physical complications are reversible consequences of the sufferer's behaviour. She needs an honest account of her current state and future prospects. Information helps the patient to define her dilemma. However, attempts to scare someone into changing are rarely successful. Osteoporosis may be less reversible although the optimal management to avoid or contain this problem is unclear.

(d) Oedema can be an alarming consequence of refeeding. This seems to occur especially in patients who abuse laxatives. The mechanism is unclear. Fluid retention may lead to weight gain of several kilogrammes over a few days, which can be extremely alarming to the sufferer. The condition is usually self-limiting but potassium sparing diuretics may be required.

Useful reviews of the physical aspects of eating disorders, including the rarer complications, can be found in Sharp \& Freeman (1993) and Treasure \& Szmuckler (1995).

\section{The brokerage of responsibility}

Characteristically the anorexia nervosa sufferer has mixed feelings about her condition. She may feel in control but precariously so. She does not want to be as she is but at the same time she is wary at best (and terrified at worst) of the prospect of change. Any change seems to carry the risk of everything getting out of control, although this may be spoken of in terms of weight gain and fatness. She feels threatened not only by others' efforts to change her but also by her own urge to eat. The management of anorexia nervosa involves the management of such mixed feelings and the proper brokerage of responsibility. In my view, it is in this matter that things most commonly go wrong.

The transaction between doctor (or other clinician) and patient can become derailed easily. The person suffering from anorexia nervosa may provoke considerable anxiety in those around her. She may push one half of her mixed feelings onto someone who is sufficiently worried about her to fulfil that role. This may be the doctor, especially in severe cases. Then doctor and patient may come together to do battle. The patient is relieved of much of the internal struggle and can simply resist the change which is being thrust upon her. On the other hand, the doctor feels obliged to fight to overcome this resistance if the patient is to be saved from herself. The most detached of clinicians may behave like a finger-wagging parent comforted by the feeling that the best is being done for a difficult patient. The whole business can come to have 
something of the character of a poker game. Each side 'ups the ante'. The patient refuses to eat at all and the doctor issues increasingly dire warnings and threats. It is possible that some of the disasters which occur in the management of anorexia nervosa can be understood as the tragic consequence of such interactions played out to the bitter end. It is better to avoid such battles (Box 2).

Another trap involves the doctor and the patient slipping into a collusion around the idea that they need to get beyond the issue of weight and eating and tackle what are usually described as the underlying problems, using the metaphor of depth. Of course, this is in a sense true. However, to act as if weight and eating are not important (or only symbolically so) is to ignore something crucial. I prefer the metaphor of entanglement of weight and eating with personal issues because it avoids placing one side in a more basic position, which seems inevitable with language employing the idea of depth. Certainly, it is possible for therapist and patient to spend hours just talking around the wider issues without touching upon the tangles which entrap the sufferer. This too is better avoided.

Assessment usually culminates in the offer of a treatment plan. The tasks of recovery are the same, whatever help is offered (Box 3). There is a need for clinician and patient to find a common language in which to discuss them. Elsewhere, I have suggested one useful way of talking but there are clearly many ways of doing this (Palmer, 1989). There may be room for compromise about some details but fudge in response to the patient's ambivalence is not helpful. It is usually best if such ambivalence is explicit rather than acted out. The patient is faced with real choices. She can opt not to change or to change only a little. Except in rare circumstances her wish not to have active treatment should be respected. If someone does not want to change it is usually best not to press the matter too

\section{Box 2. Management of mixed feelings}

Remember that the dilemma belongs to the patient

Give the patient information

Offer the patient ways forward and out of her disorder

Don't fudge the issues

Allow the patient real choice about whether to try to change

Remain in touch even if help is initially rejected hard, but rather to remain in touch and keep the matter under review and the option of treatment open. People who are not chased rarely run away and often come to accept help when they feel less panicky about the prospect.

\section{Treatment}

The treatment of anorexia nervosa is rationally directed at all three tasks of recovery (see Box 3). A treatment package that omits attention to one or more task is unlikely to be optimal. This is an assertion based on principle and experience. It is unfortunately the case that there is only a limited base of relevant treatment trials. Furthermore much of the evidence that exists relates mainly to the promotion of weight gain (task one) and is shortterm in a disorder which is usually chronic and notoriously subject to relapse. There is no evidence that drug treatments are helpful in changing weight or the core psychopathology of anorexia nervosa. They may have some role in the treatment of some symptoms (e.g. metoclopramide for bloating) and comorbidity.

\section{Task one}

If the anorexia nervosa sufferer is to eat more and gain weight she must overcome the feeling that everything will get out of control if she does so. Treatment needs to promote a situation in which she can feel safe enough to do this. For the majority of sufferers, out-patient contact suffices for this (Crisp et al, 1991). It is difficult to be sure which characteristics of this contact promote such a sense of safety. The research literature does not help much. I believe that the patient needs consistent and regular conversation with someone who seems to be confident and recognises the nature of her dilemma. Simply feeling understood may sometimes be enough to shift the balance in favour of change.

The sessions need to be predictable. Weekly meetings are usual to start with. Regular weighing by the therapist, diary keeping, encouraging a pattern to eating and advice about what to eat are further techniques. The last of these can come to be overemphasised. The patient may try to lead the conversation into prolonged negotiation about what to eat because of her anxieties. The therapist needs to give advice and structure but will often need also to edge the conversation toward more direct discussion of these anxieties and away from 
Box 3. The tasks of recovery

However it is achieved, recovery from anorexia nervosa may be thought of as the accomplishment of three tasks:

(i) Restoration of a weight that is normal, healthy and sustainable and a regular pattern of eating. This weight is usually an average one (BMI 18-25). People who have been obese may need to stabilise at a higher weight if they are to live without undue eating restraint

(ii) Disentanglement of ideas about weight and eating from wider personal issues such as self-esteem

(iii) Making progress with regard to such wider issues and problems. Problems of living and so on are seldom if ever fully 'sorted out' but once freed from the entanglement with weight and eating, life can move on again

the details of diet. The skill lies in using the particular situation as a way of looking with the patient at the links with wider personal issues.

Detailed dietary advice, perhaps from a dietician, sometimes has a place. However, it is usually robust common sense about diet which is lacking in the patient's thinking. Most of the difficulties which arise are of a kind that require psychological rather than dietetic expertise. What is required is a mixed diet which is increased at a pace which is manageable for the patient. It is sensible to give vitamin supplements to chronically malnourished subjects.

Weight gain that is faster than a kilogramme, or at most two, per week is too fast once initial dehydration is rectified. It cannot be sustained and is likely be frightening. It raises the possibility that the patient is bingeing. Is she stuffing herself in the hope of being let off some perceived hook? Families may need to be advised against constantly nagging the sufferer or tempting her to eat with favourite foods.

Weight gain that is slow or absent in the face of apparently adequate intake raises the question of the hidden use of vomiting, laxatives or diuretics. It is best to raise this suspicion and discuss the matter openly. It may be best to encourage the patient to eat only what she is willing to retain. Most patients can give up these behaviours when they resolve to do so. High doses of laxatives, for example, over 30 senna per day, are best tailed off in a stepwise fashion over a month or so. Repeated monitoring of urea and electrolytes is indicated in an unstable situation.
Some treatment plans seek to keep the supervision and promotion of weight gain separate from the exploration of wider personal issues. The patient might be provided with what amounts to two therapists, one who talks about eating and weight and one who talks about other matters in a more traditionally psychotherapeutic way. While I am sure that this can work if it is done with confidence, I see little advantage in it in the outpatient setting. It can promote splitting and furthermore may miss the little opportunities to explore the all important links between these two sets of issues which the one-therapist model allows.

\section{In-patient treatment}

In usual psychiatric practice, perhaps a third of sufferers will not make any progress with outpatient treatment alone. The patient feels stuck and too unsafe to allow herself to change. In these circumstances, it will be necessary to consider offering a more intensive treatment. This usually means hospital admission, although special day programmes are being developed and may be an alternative option (Freeman \& Newton, 1992). The patient herself will be faced with the difficult choice of whether or not to accept admission. Only in exceptional circumstances should the adult patient be relieved of this decision. Indeed, the more the whole undertaking is discussed and the more the patient commits herself to the treatment plan before admission, the more likely things are to go smoothly after admission.

\section{Indications for in-patient treatment}

Admission to hospital should be considered under the following circumstances:

(a) If the physical status of the patient is such as to cause immediate danger to life.

(b) If suicide risk or comorbidity warrants admission.

(c) If no adequate out-patient treatment is available.

(d) If the patient has failed to progress with appropriate out-patient help.

Compulsory admission under a section of the Mental Health Act can be justified in either the first or second circumstance. However, detention for the treatment of anorexia nervosa alone is indicated only very rarely. It should usually be considered only in relation to admission to a specialist service and then in a small minority of cases.

Admissions which arise out of panic are unlikely to work whether it is the patient, her family or the doctor who is panicking. A previously abstinent 
sufferer may panic if she starts to binge. Admission may be appropriate to help with this panic but often the patient's motivation does not outlast the containment of the symptom. The doctor and family may panic if there is a rapid deterioration in the patient's physical state. If there is real physical danger, admission for stabilisation under a physician may be preferable to a psychiatric admission where the longer term aims have been inadequately agreed. The confidence and consistency of care which are necessary for successful inpatient treatment can be achieved on an occasional, or one off, basis but undoubtedly are easier to contrive within a specialist unit (Box 4).

\section{Tasks two and three}

The more specific aspects of psychotherapeutic treatment aimed at helping with tasks two and three are usually best delivered in a one to one therapy session, on at least a weekly basis. When in-patient care is involved, it is ideal if the same therapist is able to see the patient before, during and after admission. However, in the context of admission there is a need to avoid undue splitting between the designated therapist and the rest of the team. The therapist needs to be seen to be a party to the in-patient enterprise and the ward staff need to be psychotherapeutically aware. Sometimes formal therapy is delayed until after inpatient weight restoration. Unfortunately, this is the pattern that has been employed in some research projects as a way of simplifying the design. They can therefore throw no light on whether this timing is optimal. My guess is that it is not.

As with any psychotherapy, sessions should be regular and predictable. The need to combine what might be called a degree of 'physicianly concern' (whatever the discipline of the therapist) with other more traditional psychotherapeutic skills means that the role demands flexibility and confidence. It also demands persistence since the whole process of recovery takes months and years even when it goes well. This can cause real problems when the patient lasts longer than the therapist, for example, if a junior doctor is assigned to that role. Nonmedical therapists need the backup of a doctor.

The style of therapy offered to anorexia nervosa patients varies. Unfortunately there is little in the literature which offers clear guidance. Provided the issue of weight and eating is not avoided, psychodynamically informed therapy and cognitive behavioural therapy (CBT) seem equally appropriate. Neither can convincingly claim superiority (Garner \& Garfinkel, 1985). The systematic evaluation of therapies which has made considerable progress
Box 4. Most in-patient regimes should have the following characteristics:

The patient should have real and informed choice about admission

The regime and its aims should be worked out before admission

These aims should include a target weight for the admission. The ultimate target should be restoration of a sustainable weight within the normal range

There should be clear control of both the lower and the upper limit of what the patient is expected to eat. The patient needs to feel that it is safe to let go some of her own internal control because the regime will stop her from overeating. All relevant staff need to understand this counterintuitive imperative and resist 'stuffing' the patient

The whole regime should be predictable and 'safe' for the patient. The use of tight behavioural regimes in which weight gain is rewarded by increasing privileges after initial deprivation have been shown to have no advantages and can feel punitive

'Fights' should be avoided by allowing the patient to leave and think again about admission. The corollary of such a policy should be that the clinician remains willing to keep in touch with the patient and to continue to offer appropriate treatment opportunities including readmission

in the field of bulimia nervosa has yet to occur to the same degree in the admittedly more difficult matter of the treatment of anorexia nervosa.

One clear finding from the first of an important series of psychotherapy research projects from the Institute of Psychiatry (Dare \& Eisler, 1995) relates to the superiority of family interventions for patients with an onset of disorder before age 18 . This study compared the experimental therapies over the year after in-patient weight restoration (Russell et al, 1987). Family therapy was compared with individual supportive psychotherapy. In a further study of adolescent out-patients (Dare et al, 1990), family therapy was found to be comparable with 'family counselling' in which the patient and her family were seen separately. In both family interventions there was an emphasis upon helping the family take responsibility for feeding the patient; an approach which may be relevant only 
for young sufferers. There was also an avoidance of blaming the family; an unfortunate feature of some family therapies. In the first study, the differences in favour of family therapy were still evident after five years.

\section{Psychiatric complications and comorbidity}

People suffering from anorexia nervosa are often distressed and even suicidal. Sometimes they show all of the features of a major depression. Such depression may limit their capacity to appraise and change their situation. It may require treatment with antidepressant medication or occasionally with ECT. The decision as to when to offer such treatments for comorbid depression is familiar territory for any psychiatrist. Of course, some degree of depression in the broadest sense is both understandable and appropriate. Indeed, anyone who is happy within her anorexia nervosa is unlikely to change.

Obsessional symptoms, alcohol or drug abuse, overdosing, self-cutting and other forms of selfharm often occur in people in treatment for anorexia nervosa. Such problems deserve assessment and intervention in their own terms and along lines which would be appropriate for other patients. The problem is to keep sight of all of the issues at once. It is rarely the case that treating a comorbid problem will lead the anorexia nervosa to remit. When present in its full form anorexia nervosa is usually the most persistent of a mixed Axis I psychopathology (Schork et al, 1994).

The most common mixture of other issues is of problems of personality and interpersonal relationships. Sometimes these amount to a diagnosable Axis II disorder. The important issue is to seek to disentangle these issues from weight and eating control and not to allow the attempt to treat the disorder to emphasise still further such entanglement. Those are the central issues of the therapy of eating disorders.

\section{Chronicity}

Anorexia nervosa is commonly a chronic disorder. Some people become firmly stuck within it. However, both clinical experience and research suggest that full recovery can occur even after many years (Herzog et al, 1992). No sufferer should be thought to have no chance to change. Nevertheless some people feel that the best they can manage is to live some sort of life within, and despite, the disorder. In such cases, the clinician needs to give support while keeping the door open for more ambitious treatment. Simply keeping in touch may be valuable (Yager, 1995).

\section{Conclusions}

The management of anorexia nervosa is never a straightforward matter of applying particular treatments. It involves the construction and use of an appropriate treatment alliance with the patient. Because of the fears and mixed feelings of the anorexia nervosa sufferer building and maintaining this alliance can be a tricky business. The most commonly missing ingredient is probably clinician confidence rather than competence. There are real problems of generalisation from the limited research findings to ordinary clinical practice. Thus, it would be inappropriate to conclude that all younger anorexia nervosa sufferers are always best treated by any family therapy, or that because there are indications that a specialist day programme may be effective attending any general day hospital would achieve the same. The clinician must decide what is 'the best buy' locally rather than seeking to apply the headline messages of research in the manner of someone consulting a cookery book. This is not to disparage the available findings but rather to suggest that the small quantity of good research limits the confidence with which the results can be appropriately generalised.

It is a conundrum of service provision whether the interests of people with eating disorders are best promoted by the creation of specialist services or by making the treatment of anorexia the task of every psychiatric team. Almost certainly some kind of compromise is optimal in which improvement of general service combines with continuing specialist services for severe cases (Eating Disorders Association, 1995). The conundrum for the general psychiatrist considering the individual patient concerns when to seek more specialist help if things are not going well. At present, the provision of special services is patchy and haphazard.

\section{References}

Crisp, A. H. (1980) Anorexia Neroosa: Let Me Be. Academic Press: London.

- Norton, K., Gowers, S., et al (1991) A controlled study of the effect of therapies aimed at adolescent and family psychopathology in anorexia nervosa. British Journal of Psychiatry, 159, 325-333. 
Dare, C., Eisler, I., Russell, G. F. M., et al (1990) The clinical and theoretical impact of a controlled trial family therapy in anorexia nervosa. Journal of Marital and Family Therapy, 16, 39-57.

— \& — (1995) Family therapy. In Handbook of Eating Disorders: Theory, Treatment and Research (eds G. Szmuckler, C. Dare \& J. Treasure), pp. 333-349. Wiley: New York.

Eating Disorders Association (1995) Eating Disorders - A Guide for Purchasing and Providing Services. Norwich: EDA.

Freeman, C. P. \& Newton, J. R. (1992) Anorexia nervosa: what treatments are effective? In Practical Problems in Clinical Psychiatry (eds K. Hawton \& P. Cowen), pp. 77-92. Oxford: Oxford University Press.

Garner, D. M. (1995) Measurement of eating disorder psychopathology. In Eating Disorders and Obesity; A Comprehensive Handbook (eds K. D. Brownell \& C. G. Fairburn), pp. 117121. New York: Guilford.

- \& Garfinkel, P. E. (1985) Handbook of Psychotherapy for Anorexia Nervosa and Bulimia. New York: Guilford Press.

Herzog, W., Deter, H-C. \& Vandereycken, W. (1992) The Long Term Course of Eating Disorders. Berlin: Springer Verlag.

Lask, B. \& Bryant-Waugh, R. (1993) Childhood-Onset Anorexia Nervosa and Related Eating Disorders. Hove: Erlbaum.

Palmer, R. L. (1989) The spring story: a way of talking about clinical eating disorder. British Review of Anorexia Nervosa and Bulimia, 3, 13-16.

Russell, G. F. M., Szmuckler, G., Dare, C., et al (1987) An evaluation of family therapy in anorexia nervosa and bulimia nervosa. Archives of General Psychiatry, 44, 1047-1056.

Schork, E. J., Eckert, E. D. \& Halmi, K. A. (1994) The relationship between psychopathology, eating disorder diagnosis and clinical outcome at 10 year follow up in anorexia nervosa. Comprehensive Psychiatry, 35, 113-123.

Sharp, C. W. \& Freeman, C. P. L. (1993) The medical complications of anorexia nervosa. British Journal of Psychiatry, 162, $452-462$.

Treasure, J. \& Szmuckler, G. (1995) Medical complications of chronic anorexia nervosa. In Handbook of Eating Disorders; Theory, Treatment and Research (eds G. Szmuckler, C. Dare \& J. Treasure), pp. 197-220. Chichester: Wiley.

Yager, J. (1995) The management of patients with intractable eating disorders. In Eating Disorders and Obesity; A Comprehensive Handbook (eds K. D. Brownell \& C. G. Fairburn), pp. 374-378. New York: Guilford.

\section{Multiple choice questions}

1 The following are well recognised physical complications of anorexia nervosa:

a Oedema on refeeding

b Hyperkalaemia

c Leucocytosis

d Hypoproteinaemia

e Osteoporosis

2 The following are in general true of anorexia nervosa sufferers:

a Most eventually recover b Comorbid major depression is rare

c Most do not wish to recover

d Treatment of depression leads to remission of the anorexia

e Most have no urge to eat

3 The following statements are generally true of the management of anorexia nervosa:

a In-patient care is almost always required

b Chlorpromazine has a specific beneficial effect

c It is useful to put an upper limit on the expectation of what the patient will eat

$d$ Anorexia nervosa is not a mental disorder within the meaning of the Mental Health Act 1983

e The faster the rate of weight gain the better

4 In general, when trying to help someone with anorexia nervosa, it is better to:

a Avoid discussing their physical state

b Avoid getting into battles with them

c Avoid involving their family

d Avoid taking over the patients' dilemma

e Avoid talking about weight

5 Research has supported the following statements:

a Strict in-patient behavioural regimes have no advantages

b Psychotherapy is not useful until after weight restoration

c Special day-treatment programmes can be helpful

d Family therapy may be superior to individual therapy for patients with an age of onset before 18

e Recovery from anorexia nervosa does not occur when the disorder has been present for more than 12 years

\begin{tabular}{|cccccccccc|}
\hline \multicolumn{2}{|c|}{ MCQ } & answers \\
1 & & 2 & & 3 & & 4 & & 5 & \\
a & T & a & T & a & F & a & F & a & T \\
b & F & b & F & b & F & b & T & b & F \\
c & F & c & F & c & T & c & F & c & T \\
d & T & d & F & d & F & d & T & d & T \\
e & T & e & F & e & F & e & F & e & F \\
\hline
\end{tabular}

